\title{
Bibliografía Anatómica Utilizada en la Formación de Médicos en Chile entre 1758 y 1840
}

\author{
Anatomical Bibliography Used in the Training of \\ Physicians in Chile Between 1758 and 1840
}

\author{
Juan Silva ${ }^{1,2}$; Claudia Araya ${ }^{1}$; Julio Cárdenas²; Andrés Salcedo ${ }^{3}$; Bárbara Mansilla ${ }^{1}$; \\ Andrea Hernández ${ }^{1}$; Pablo Rodríguez ${ }^{1}$; Matías Salazar ${ }^{1}$; Rodrigo Caracuel $^{1} \&$ Mario Espinoza $^{1}$
}

SILVA, J.; ARAYA, C.; CÁRDENAS, J.; SALCEDO, A.; MANSILlA, B.; HERNÁNDEZ, A.; RODRÍGUEZ, P.; SALAZAR, M.; CARACUEL, R. \& ESPINOZA, M. Bibliografía anatómica utilizada en la formación de médicos en Chile entre 1758 y 1840 . Int. J. Morphol., 37(3):938-946, 2019.

RESUMEN: El estudio de la enseñanza de la medicina, desde una perspectiva histórica, es importante para explicar el desarrollo de esta profesión. Considerando el valor de los libros en el currículo universitario su estudio permite comprender la influencia sociopolítica del momento histórico. El objetivo de este trabajo fue determinar y analizar la primera bibliografía utilizada en la formación de médicos en Chile entre 1758 y 1840 (desde la fundación de la Universidad de San Felipe a la muerte del Dr. Pedro Morán). A través de una investigación histórica se logró encontrar los ocho libros de textos utilizados durante este periodo. De ellos, se realizó un análisis descriptivo que abarcó la identificación del autor, el título de la obra, el año, idioma y lugar de publicación. Además, se clasificaron según extensión y enfoque. También se realizó un análisis cuantitativo para determinar la cantidad de información que presentan estos libros en comparación con la literatura moderna. Los resultados se ordenaron en tablas y gráficos para su posterior análisis. Los textos encontrados fueron principalmente editados en España y en idioma español $(62,5 \%)$, aunque una parte importante fueron editados en Francia y en francés $(37,5 \%)$. Mayoritariamente se utilizó bibliografía de autores franceses (50\%), aunque también se incluyeron libros de autores españoles $(37,5 \%)$ y alemanes $(12,5 \%)$. Con respecto a la extensión de los textos, tanto los libros de mediana y gran extensión estuvieron representados de igual forma $(37,5 \%)$ y los compendios representaron un porcentaje menor (12,5\%). Este trabajo es un aporte a la comprensión del origen de la formación de médicos en Chile y ayuda a comprender el nacimiento de la identidad profesional, que ha sido de gran influencia en la historia político - social de Chile, describiendo la bibliografía que marco el nacimiento de la Historia de la Anatomía en nuestro país.

PALABRAS CLAVE: Historia de la Anatomía; Historia de la medicina; Historia de Chile; Bibliografía anatómica; Universidad de San Felipe; Instituto Nacional.

\section{INTRODUCCIÓN}

Se decide estudiar este periodo de la historia para abarcar la bibliografía anatómica bajo el influjo de los primeros profesores médicos, que formaron parte de la primera y segunda escuela de medicina del país, iniciando este estudio en el año de 1758 con el inicio de la formación médica en la Universidad de San Felipe (USF) y terminando en el año 1840 con la muerte de Pedro Morán.

En Chile durante el periodo comprendido entre los años 1758 y 1840 se formaron médicos y cirujanos en dos instituciones formales; la USF y el Instituto Nacional (IN). Estos estudios se vieron complementados de manera infor- mal, por cursos dictados en el Hospital San Juan de Dios (HSJD) y en cursos privados dictados por el Dr. Pedro Morán (entre 1826 y 1833).

La USF es fundada por Cédula Real de San Ildefonso (Madrid) con fecha 28 de julio de 1738. Pero recién en 1756 fueron nombrados los diez catedráticos que dictarían los cursos de Filosofía, Teología, Leyes, Matemáticas y Medicina (Gay, 2007; Serrano, 2016). En Prima Medicina se nombró como profesor al Dr. Domingo Nevin McHugh (irlandés 1722 - 1770) (Medina, 1928). Dos años más tarde, el 9 de enero de 1758, comenzaron las clases oficialmente en

\footnotetext{
${ }^{1}$ Facultad de Medicina, Universidad San Sebastian, Santiago, Chile.

${ }^{2}$ Departamento de Anatomía y Medicina Legal, Facultad de Medicina, Universidad de Chile, Santiago, Chile.

${ }^{3}$ Facultad de Odontología, Universidad San Sebastian, Santiago, Chile.
} 
SILVA, J.; ARAYA, C.; CÁRDENAS, J.; SALCEDO, A.; MANSILlA, B.; HERNÁNDEZ, A.; RODRÍGUEZ, P.; SALAZAR, M.; CARACUEL, R. \& ESPINOZA, M. Bibliografía anatómica utilizada en la formación de médicos en Chile entre 1758 y 1840. Int. J. Morphol., 37(3):938-946, 2019.

esta casa de estudios (Grossi, 1895; Barros - Arana, 2001; Gay). En el caso de medicina y según la pragmática de Felipe III, la carrera duraría cuatro años y su horario de clases consideraba una hora de lectura diaria de las obras de Hipócrates, Galeno y Avicena y posteriormente media hora de discusión (Grossi; Laval, 1958). Normalmente los cursos se dictaban en latín y la enseñanza era principalmente memorística, con estricto ajuste a textos o dictados (Hernández, 1934; Mellafe et al, 1992; Cruz-Coke, 1995; Barros - Arana). Durante el tiempo que se dictó la carrera de Medicina en la USF, se trató que los estudiantes utilizaran libros impresos como se hacía en España y los libros que se indicaban con este objetivo se encontraban en Chile, pero solo en las bibliotecas de los catedráticos (Mellafe et al).

Durante sus primeros años la USF, no tuvo una biblioteca propia. Las obras de medicina que conocieron los médicos coloniales fueron más bien escasas y de alto precio, cuando se lograban hallar en el comercio. Inclusive durante muchos años descubrir un buen libro de estudios era poco menos que descubrir un tesoro, pero si no estaba traducido al español, o en último término al francés, más valía ni pensar en él (Ferrer, 1904; Sierra, 1934; Barros-Arana).

Paralelo a la educación formal en las aulas de la USF, el Dr. Pedro Manuel Chaparro (chileno 1745 - 1811), fraile perteneciente a la orden hospitalaria de San Juan de Dios y primer estudiante universitario en Chile que obtuvo el grado de Doctor en Medicina, se dedicó en forma extraoficial a la formación práctica de médicos y cirujanos. Para Chaparro, la enseñanza de la anatomía era tan fundamental que en la práctica exigía a los estudiantes su estudio durante cinco años y aconsejaba que en los dos primeros se enseñase con el Compendio Anatómico de Lorenzo Heister (Fig. 1a), traducido del alemán por el cirujano español Andres García Vázquez (español 1693 - ?) y la Anatomía Completa del Hombre de Martín Martínez (Fig. 1b), publicados en 1755 y en 1764 respectivamente (Medina; Laval, 1964; Cruz-Coke; Laval \& Duarte, 2016).

En 1813, durante el gobierno del General Jose Miguel Carrera (chileno 1785 - 1821), se funda en la ciudad de Santiago el "Instituto Nacional, Literario, Económico, Civil y Eclesiástico del Estado". Este establecimiento fue concebido originalmente como la institución modelo que serviría de matriz para la educación pública. Todos los niveles educativos, todos los campos del saber, su cultivo y su aplicación, estaban comprendidos en él, incluyendo los estudios superiores de teología, ciencias naturales, derecho y medicina (Serrano).

En el expediente presentado en 1813 por la Junta de Educación, figura el "Reglamento y Plan de Estudios del IN". En este se propone la creación de 3 cátedras relaciona-
ANATOMIA COMPLETA DEL HOMBRE, CON TODOS LOS HALLAZGOS, NUEVAS DOCTRINAS, Y OBSERVACIONES RARAS

HASTA EL. TIEMPO PRESENTE, $\checkmark$ MUCHAS ADVERTENCIAS NECESSARIA\&

PARA LA CIRUGIA:

SEGUN EL METHODO CON QUEE SE EXPLICA EN NUESTRO THEATRO DE MADRID.

TRADUCIDO DE LA LENGUA LATINA, fegun la ultima, y mas correpta edícion del Autor, c̀ ilustrado con Eftampas finas,

POR DON ANDRES GARCIAVAZQUEZ, Cirujano de Familia de S.M. Fundador, y actual Director del Real Colcgio de San Fernando, de Cirujatios de Madrid.

CON PRIVILEGIO: En Madrid, por los Herederos de Don Migapil Francifico Rodriguez. Ańo de 1755 .

TI Se ballarì con los quatro Tomos de la Cirugia Completa, y tres de Inframciones Medicas, yedicina Praltica del mifmo Awtor, en la Lonja e Papel de Jofoph de Sierra, freste de las Gradas de $S_{\text {ant }}$ Phelipe; y tambien Las obras de Genga, $)$ del famojo Inglis Samwil Sharp:

\section{$\begin{array}{lll}P & O & R\end{array}$ \\ EL DOCTOR DON MARTIN MARTINEZ,} Medico de Camara de Ju Magefad, Socio, y Ex-Prefudenta de La Regia Sociedad de Sevilla, Profeffor Publica de Avarouria, $y$ Examinador que fue do fo Roal Proto-Medicato. emen: CON LIC ENCIA:

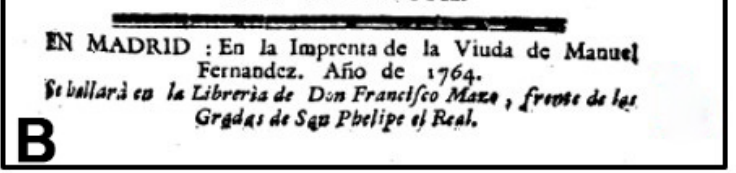

Fig. 1. Portadas de los libros de texto, en "a" de Heister, L. (1755) y en "b" de Martínez, M. (1764), respectivamente. 
das con la enseñanza de la medicina (cirugía, medicina teórica y medicina práctica). Además, se indica que los libros a considerar en anatomía, son el Compendio de Anatomía de Juan de Dios López (Fig. 2a) y el Curso Completo de Anatomía del Cuerpo Humano de Jaime Bonells \& Ignacio Lacaba (Fig. 2b), publicados en 1750 y 1820, respectivamente (Laval).

Pero no sería hasta 1833 que se diera inicio oficialmente, al primer curso de medicina en el IN. Su programa oficial establecía un periodo de 6 años de estudios e incluía las siguientes asignaturas: anatomía, fisiología, higiene, principios y prácticas de la medicina, materia médica, medicina clínica, cirugía, obstetricia, y enfermedades de los niños (Cruz-Coke; Laval, 2011).

En este primer curso de medicina del IN, el profesorado original estaba compuesto por los miembros del Tribunal del Protomedicato; Dr. William Cunningham Blest Maiben, (irlandés 1800 - 1884), Dr. Pedro Morán (chileno 1771 - 1840) y el farmacéutico José Vicente Bustillos (chileno 1800 - 1873). De estos, el Dr. Pedro Morán fue nombrado Profesor de Anatomía (Grossi; Cruz-Coke). A él le tocó inaugurar en 1833 el anfiteatro anatómico y en dicha oportunidad indicó en su discurso inaugural: “(...) principiaron las lecciones de ambos cursos (anatomía y medicina), bajo los sistemas más dignos de las luces del siglo. Para el anatómico, puesto bajo mis débiles luces, tome por tipo a los señores Bichat (Tratado de Anatomía, 1812) y Maygrier (Nuevo Manual de Anatomía, 1820)." (Laval; Orrego, 1922; Flores, 1933). Este último texto fue traducido del francés por el médico y cirujano Manuel Hurtado de Mendoza (español 1783 - 1849). Las portadas de ambos libros se observan en las Figuras $3 a$ y b, respectivamente.

Además también se sabe que Morán seguía en sus clases el texto de Bonells \& Lacaba (Salas, 1894) y que los alumnos estudiaban en textos importados, ingleses, franceses y traducciones españolas tales como el Tratado de Anatomía Descriptiva de Bichat (Cárdenas, 2017).

Con respecto a la apertura de la Escuela médica chilena, se indica que tal era la escasez de textos de enseñanza en esa época (1833) que de anatomía solo se conocían las obras de Bayle (Compendio de Anatomía, 1823) (Fig. 4a) y Maygrier (Semir, 1860). Con respecto a este último libro, existen registros de la biblioteca del IN que indican que en 1826 existía una copia en sus estanterías (Amunategui, 1889).

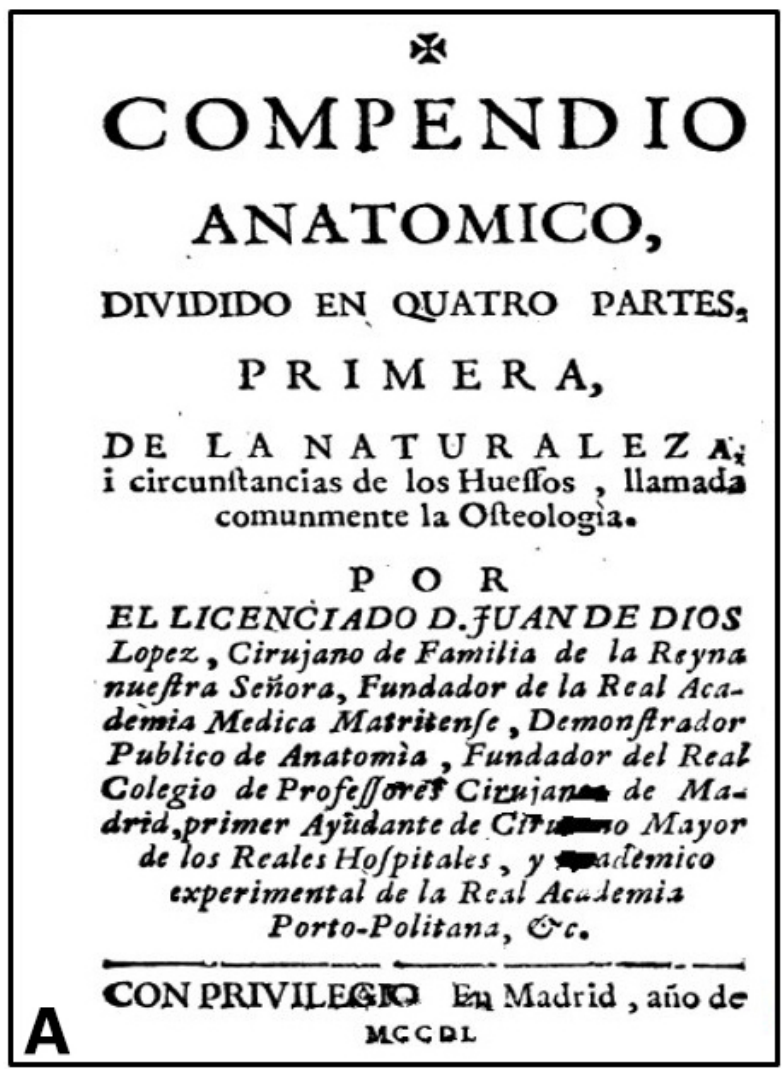

CURSO COMPLETO DE ANATOMIA DEL CUERPO HUMANO P O R
EL DOCTOR DON JAIME BONELLS, Y POR
EL LICENCIADO DON IGNACIO LACABA.

OSTEOLOGIA TMIOLOGIA.

SEGUNDA EDICION.

MADRID:

EN LA IMPRENTA QUE FUE DE FUENTENEBRO. 1820 .

Fig. 2. Portadas de los libros de texto, en "a" de Lopez, J. (1750) y en "b" de Bonnels, J. \& Lacaba, I. (1820), respectivamente. 


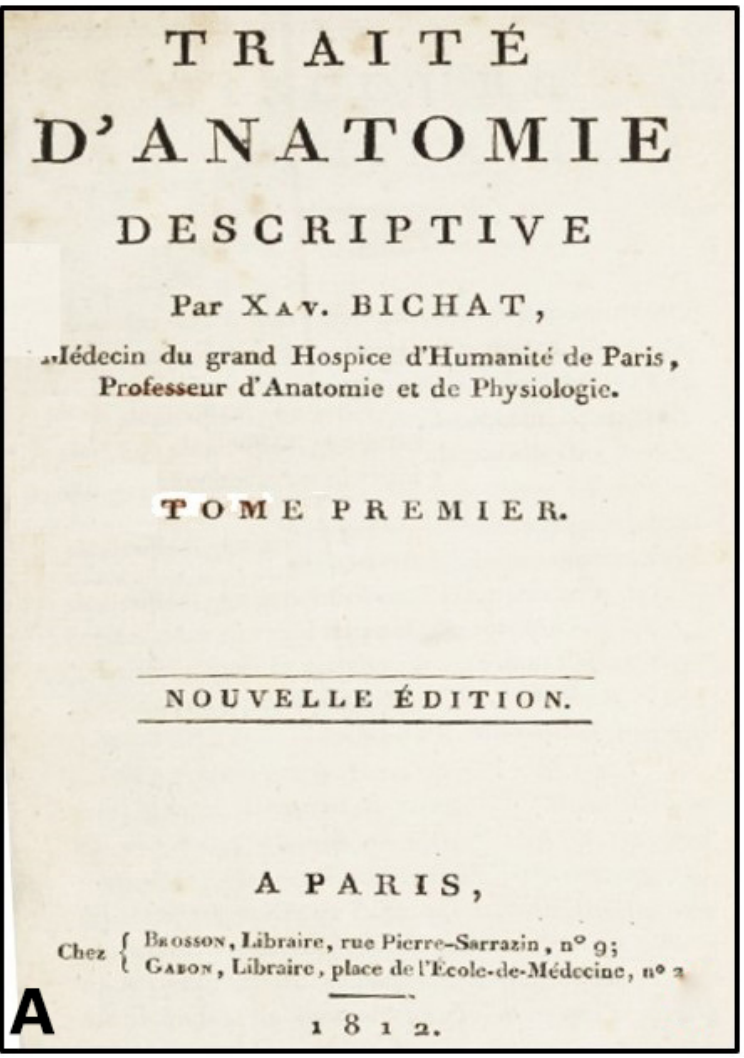

Fig. 3. Portadas de los libros de texto, en "a" de Bichat, X. (1812) y en "b" de Maygrier, J. (1820), respectivamente.

PETIT

\section{MANUEL D'ANATOMIE DESCRIPTIVE,}

ov

DESCRIPTION SUCCHNCTE DE TOUS LES ORGANES DQE

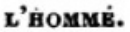

Par A. L. J. BAYLE,

Docteur en médecine de la Faculté de Paris, Médecin at taché a la Maison royale de Charenton, Membra de l'A thénée de médecine, etc.

PARIS et MONTPELLIER, CHEZ GABON BT COMPAGNIB, LIBRAIRES. A 1823 .

\section{NUEVO MANUAL}

DE ANATOMIA,

O TRATADO METÓdICO Y RAZONADO SOBRE

EL MODO DE PREPARAR TODAS LAS PARTES DE TA ANATOMFA, SEGUIDO DE UNA DESCRIPCION COMPLETA DE ESTAS MISMAS PARTES;

POR J. P. MAYGRIER,

Doctor en medicina de la facultad de París, catedrático de anatomía y de fisiología, de partos, de enfermedades de mugeres $y$ de níios; miembro de la sociedad médica de emulacion y de la dicina de la cindad de Lieja y de Tolosa de la de Ciecias de la de Ciencias de Macon, de Marsella, \&cc. \&c.

CUARTA EDICION,

REVISTA, CORREGIDA Y AUMENTADA. TRADUCIDA DEL FRANCES AL ESPANOL,

POR DON MANUEL HURTADO DE MENDOZA, doctor en medicina y en cirugia-medica, socio corresponsal de las academias médicas nacionales de Madrid, y meilico-práctica de Barcelona; de la socie. dad médico-quirúrgica de Cádiz, y de la sociedad y academia general de ciencias de Córdova y su reino; de la facultad de medicina de Paris, y de la sociedad médica de emulacion establecida en su seno; del real Ateneo de medicina, del ctrculo médico ó academia de medicina, y de la sociedad de medicina práctica.de Burdeos, Mompeller y de Marsella; de ia real acadernia de ciencias fisicas y médicas de Orleans; de la academia imperial Josefina de Viena, de la sociedad médico-quirúrgica de Filadelfia, ઉc. ખc.

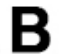

MADRID. IMPRENTA CALLE DE LA GREDA. 1820.

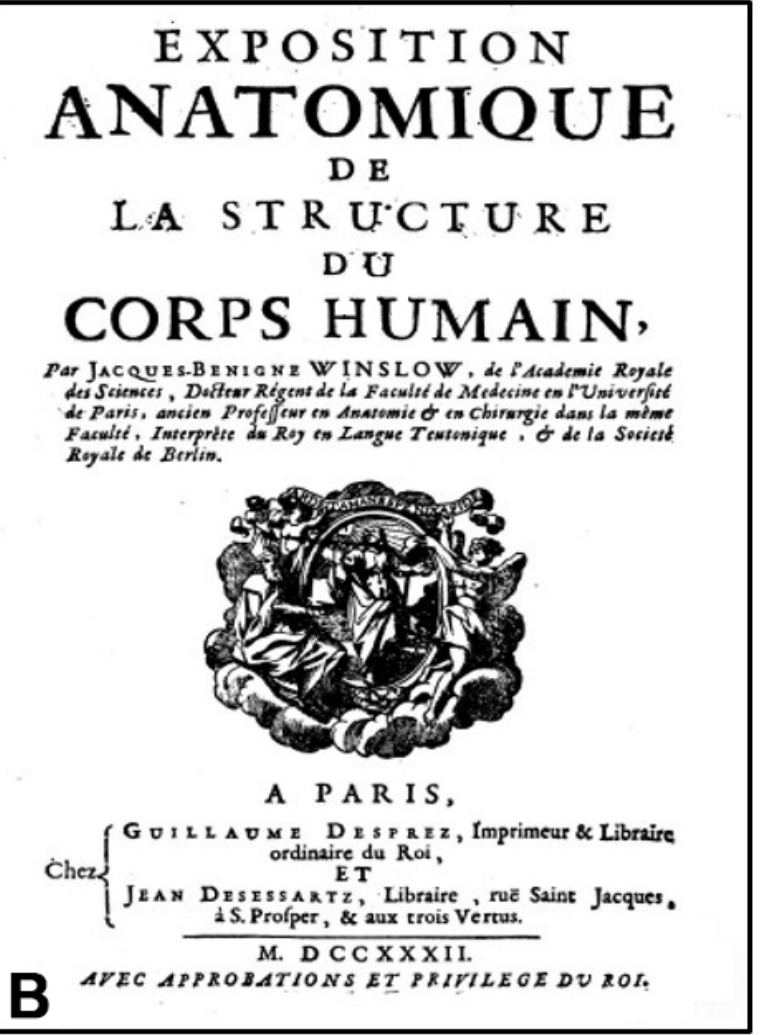

Fig. 4. Portadas de los libros de texto, en "a" de Bayle, A. (1823) y en "b" de Winslow, J. (1812), respectivamente. 
Esta escasez de libros preocupaba hasta al Presidente del Gobierno, Don Jose Joaquín Prieto Vial (chileno 1786 1854), en consecuencia, el Ministro Manuel Montt Torres (chileno 1809 - 1880), indicaba que “(...) el curso (de medicina), tenía el número de profesores necesarios, pero carecía de libros de enseñanza y consideraba que era una de las causas que retardaban la enseñanza de las ciencias médicas", debido a esto solicitó a Antonio Varas de la Barra (chileno 1817 - 1886) que “(...) reuniera a los profesores del ramo y se determinara de manera precisa, cuáles eran los libros que deben adoptarse en cada curso y en cada caso que fuere preciso, pedirlos fuera del país" (Campos, 1960). Con respecto a esta situación podemos indicar que antes de 1833 , en las escasas librerías y pequeñas bibliotecas privadas de Santiago, se podían obtener como libros de consulta, a los siguientes autores: Bayle, Bonells \& Lacaba, Juan de Dios Lopez y Winslow (Fig. 4b) (Ferrer). Este último texto (Exposición anatómica de la estructura del cuerpo humano, 1732) ya había sido recomendado, junto a Bonells \& Lacaba, como adecuados y necesarios en la formación anatómica en un informe enviado a la Junta de Educación por el Dr. Gregorio Paredes (peruano 1778 - 1839) (Salas).

Junto a la muerte del Dr. Pedro Morán (1840), hemos querido dar término a esta revisión de la bibliografía anatómica en la Historia de la Medicina de Chile. En 1841 es nombrado Profesor de Anatomía el médico francés Dr. Francisco Julio Lafargue (Laval, 2012), quien por su origen, formación y experiencia, dará nuevo rumbo y bríos a la historia de esta disciplina en Chile, trayendo nuevos textos desde Europa.

El objetivo de este trabajo fue, analizar en forma descriptiva y cuantitativa el primer grupo de libros de texto utilizados en el estudio de la anatomía de los médicos formados en Chile entre 1758 y 1840.

\section{MATERIAL Y MÉTODO}

Se realizó una búsqueda en diversos y múltiples repositorios académicos digitales, en búsqueda de los libros de texto mencionados en el análisis histórico del periodo comprendido entre los años 1758 y 1840. Estos libros se ordenan alfabéticamente a continuación:

1. Bayle, A. Petit manuel d'anatomie descriptive, description succincte de tous les organes de l'homme. Imprimerie de Gueffier. Paris, 1823.

2. Bichat, X. Traité d'anatomie descriptive. Nueva ed. Imprimerie de Cosson, Paris, 1812.
3. Bonells, J; Lacaba, I. Curso completo de anatomía del cuerpo humano. 2da ed. Imprenta que fue de Fuentenebro. Madrid, 1820.

4. Heister, L. Compendio anatómico, que brevissimamente comprehende, y explica esta ciencia. Imprenta de los herederos de Don Miguel Francisco Rodriguez, Madrid, 1755.

5. López, J. Compendio anatómico dividido en quatro partes. Imprenta del Consejo de Indias, Madrid, 1785.

6. Martínez, M. Anatomía completa del hombre con todos los hallazgos, nuevas doctrinas y observaciones raras hasta el tiempo presente y muchas advertencias necessarias para la cirugía: según el méthodo con que se explica en nuestro theatro de Madrid. Imprenta de la Viuda de Manuel Fernandez, Madrid, 1764.

7. Maygrier, J. Nuevo manual de anatomía o tratado metódico y razonado sobre el modo de preparar todas las partes de la anatomía, seguido de una descripción completa de estas mismas partes. Traducción de la 4ta ed. francesa. Imprenta calle de la Greda, Madrid, 1820.

8. Winslow, J. Exposition anatomique de la structure du corps humain. Editorial Imprenta de Guillaume Desprez. Paris, 1732.

Estos archivos se imprimieron y encuadernaron, lo que permitió su manipulación física facilitando un mejor análisis posterior de cada libro:

El Análisis descriptivo comprendió la identificación del autor, el título de la obra, el año y lugar de publicación e idioma, tanto de la primera edición como de las posteriores (si las hubiera). También se determinó el año, lugar e idioma de las traducciones de la obra (si las hubiera). Por último se clasificaron estos textos según extensión (compendio, texto o tratado) y según enfoque (descriptivo, topográfico o una mezcla de ambos).

El Análisis cuantitativo comprendió la determinación de la cantidad de información contenida en cada texto. Para esto se diseñó y calculo un índice que denominamos; Índice de Cantidad de Información (ICI) que permitió cuantificar la información contenida en cada libro.

Este ICI contempla las siguientes variables: cantidad total de páginas del libro (P) (para éste cálculo no se consideraron las páginas de; título, índice, introducción, prólogos, prefacios, imágenes, glosario y otros que no incluyeran contenido disciplinar anatómico) y cantidad total de palabras por página $(\mathrm{p})$. 
Además, para que el resultado de ICI nos permitiera la comparación entre libros, se consideró en la fórmula matemática, una constante. Para definir esta constante, se utilizaron como parámetros los siguientes textos; Anatomía con Orientación Clínica (7ª ed., 2013) de Moore, K; Dailey, A. y Agur, A. y Gray Anatomía para Estudiantes ( $3^{\mathrm{a}}$ ed. 2015) de Drake, R., Vogl, A. y Mitchell, A. De estos 2 libros se determinaron "P" y "p", estos números se multiplicaron y promediaron. A este promedio simple se le denominó MD y fue utilizado como constante $(289822,5)$.

Las variables de cada texto ("P" y "p") y la constante (MD) ahora, se relacionaron a través de la siguiente formula que denominamos ICI comparado: $\operatorname{ICI}(\mathrm{c})=(\mathrm{P} x \mathrm{p}) /$ MD.

Este número $\mathrm{ICI}(\mathrm{c})$ nos permitió realizar la comparación entre los libros de la primera bibliografía y los libros utilizados en la constante. De esta manera, aquellos textos que su ICI(c) fuera mayor a 1.0, corresponderían a libros con mayor cantidad de información que el promedio de los libros que conformaron la constante MD y aquellos con un ICI menor a 1.0, libros que presentan menor cantidad de información.

\section{RESULTADOS}

Los resultados del análisis descriptivo de los libros que constituyeron las primeras referencias bibliográficas en la formación anatómica de médicos en Chile durante el periodo comprendido entre 1758 y 1840 se resumen en la Tabla I y en las Figuras 5 y 6.

El análisis cuantitativo de los libros que conformaron este primer canon bibliográfico anatómico y que fue utilizado en la formación de los primeros médicos en Chile, se resume en la Tabla II.

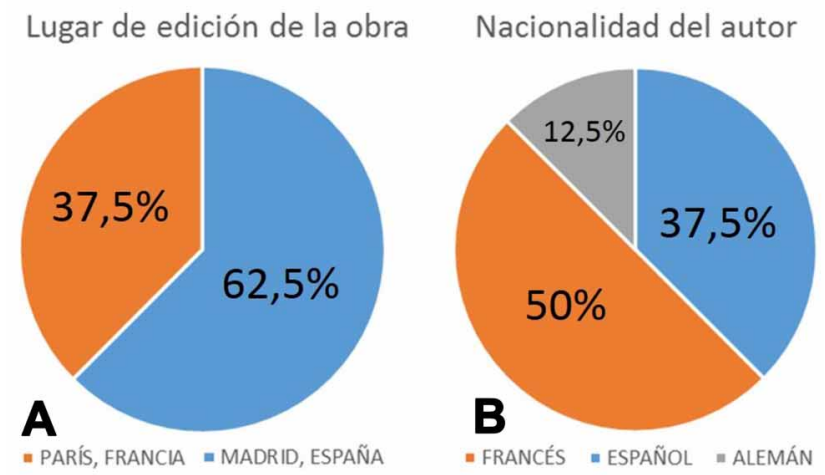

Fig. 5. Gráficos de distribución tanto del Lugar de edición de la obra la (a) como de la Nacionalidad del autor (b).
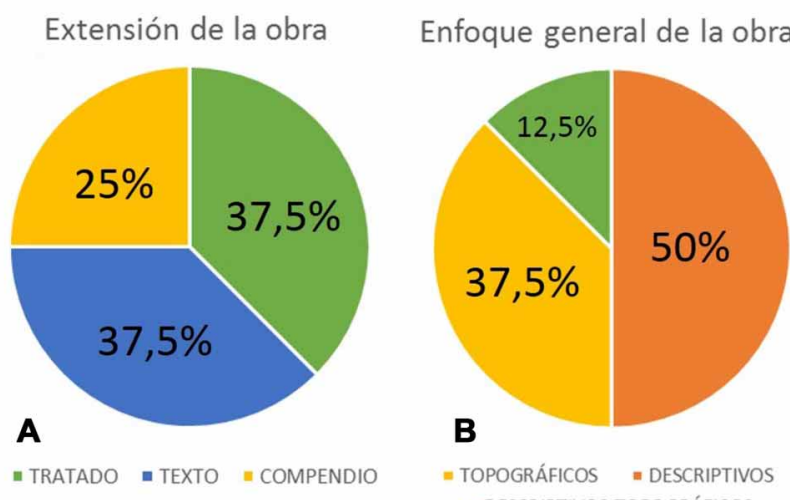

Fig. 6. Gráficos de distribución tanto de la Extensión de la obra (a) como del Enfoque general de la obra (b).

También se elaboró una línea de tiempo (Fig. 7) que permitió el análisis temporal de la aparición de los textos de este primer canon bibliográfico, en la literatura especializada en la Historia de la Medicina Chilena. Este recurso, permite evidenciar la relación de los libros con los momentos históricos que vivió Chile en el periodo analizado.

\section{CONCLUSIONES}

Del análisis descriptivo: Como lo indica la Tabla I y se grafica en la Figura 5a, cinco de estos textos fueron editados en España $(62,5 \%)$ y en idioma español. De estos textos, tres son de autores españoles $(60 \%)$, uno es de autor alemán, traducido al español (20\%) y uno de autor francés, traducido al español $(20 \%)$. Los restantes tres textos, son de origen e idioma francés $(37,5 \%)$.

La nacionalidad de los autores aparece en la Tabla I y se grafica en la Figura 5b. Así, el $50 \%$ de ellos es francés, el $37,5 \%$ es español y el $12,5 \%$ es alemán.

Su extensión (Tabla I y Fig. 6a) se distribuyó de forma semejante entre los textos de mediana extensión (37,5 $\%)$ y los textos de mayor extensión o tratados $(37,5 \%)$. En menor cantidad aparecen los textos de pequeña extensión o compendios $(25 \%)$,

Su enfoque anatómico (Tabla I y Fig. 6b) se distribuyó entre descriptivos $(50 \%)$, topográficos $(37,5 \%)$ y una mezcla de ambos $(12,5 \%)$.

Sus años de edición (Tabla I) abarcaron desde 1728 y hasta 1826 (pese a que existen ediciones en idioma ingles del texto de Maygrier posteriores a esa fecha, de estas versiones no existen referencias históricas en Chile). 
Tabla I. Tabla que resume los resultados del análisis descriptivo de los libros de textos utilizados en la formación anatómica de médicos en Chile entre 1758 - 1840. LUGAR: ciudad y país donde se publicaron los libros de textos. AÑOS: años en que se publicaron las distintas ediciones de los libros de textos. EXTENSIÓN: según clasificación y su propia definición. ENFOQUE: característica general de la visión de la anatomía que el autor dio a su obra.

\begin{tabular}{|c|c|c|c|c|c|c|}
\hline Autor & Título & Lugar & Idi oma & Años & Extensión & Enfoque \\
\hline Bayle, A. & $\begin{array}{ll}\text { Petit manuel d'anatomie } \\
\text { descriptive ou } & \text { description } \\
\text { succinte de tous les } & \text { organes de } \\
\text { l'hommè } & \end{array}$ & París, Francia & Francés & $\begin{array}{ll}1 \text { a ed., } 1823 ; & 2 \mathrm{a} \\
\text { ed., } 1824 ; & 3 \mathrm{a} \\
\text { ed., } 1826 . & \end{array}$ & Compendio & $\begin{array}{l}\text { Descriptivo y } \\
\text { Topográfico }\end{array}$ \\
\hline $\begin{array}{l}\text { Bichat, X.; } \\
\text { Buisson, M.; } \\
\text { Roux, P. }\end{array}$ & Traite d'anatomie descriptive & París, Francia & Francés & $\begin{array}{l}\text { Tomo } 1,1812 ; \text { Tomo } \\
2 \text { al } 5,1829 .\end{array}$ & Tratado & Descriptivo \\
\hline $\begin{array}{l}\text { B onells, J.; } \\
\text { Lacaba, I. }\end{array}$ & $\begin{array}{l}\text { Curso completo de anatomía del } \\
\text { cuerpo humano }\end{array}$ & Madrid, España & Español & $\begin{array}{l}\text { 1a ed., 1796; } \\
\text { ed., } 1820 .\end{array}$ & Tratado & Descriptivo \\
\hline Heister, L. & $\begin{array}{l}\text { Compendio anatómico, que } \\
\text { brevis simamente comprehende y } \\
\text { explica esta ciencia }\end{array}$ & Madrid, España & Español & 1755 & Compendio & Topográfico \\
\hline Lopez, J. & Compendio anatómico & Madrid, España & Español & $\begin{array}{c}\text { Tomo 1, 1750; } \\
\text { Tomo 2, 1751; } \\
\text { Tomos } 3 \text { y 4; } 1767\end{array}$ & Texto & Descriptivo \\
\hline Martinez, M. & $\begin{array}{l}\text { Anatomía completa del hombre, } \\
\text { con to dos los hallazgos, nuevas } \\
\text { doctrinas y observaciones raras } \\
\text { hasta el tiempo presente, y } \\
\text { muchas advertencias necessarias } \\
\text { para la cirugía: según el methodo } \\
\text { con que se explica en nuestro } \\
\text { theatro de Madrid. }\end{array}$ & Madrid, España & Español & $\begin{array}{c}1728 \\
\text { Reimpresiones en } \\
1745,1764,1775 \mathrm{y} \\
1788 .\end{array}$ & Texto & Topográfico \\
\hline Maygrier, J. & $\begin{array}{l}\text { Nuevo manual de anatomía, o } \\
\text { tratado metódico y razonado } \\
\text { sobre el modo de preparar todas } \\
\text { las partes de la anatomía, } \\
\text { seguido de una descripción } \\
\text { completa de estas mismas partes }\end{array}$ & $\begin{array}{l}\text { París, Francia } \\
\text { Madrid, España } \\
\text { New York, USA } \\
\text { Londres, UK }\end{array}$ & $\begin{array}{l}\text { Francés } \\
\text { Español } \\
\text { Inglés }\end{array}$ & $\begin{array}{c}\text { Francés: } \\
\text { la ed., 1807; } \quad \text { 2a } \\
\text { ed. 1811; } \quad 3 \mathrm{a} \\
\text { ed., 1813; } \quad 4 \mathrm{a} \\
\text { ed., 1818. Español: } \\
\text { 1820. Ingles } \\
\text { USA: 1832. } \\
\text { Ingles UK: } 1839 .\end{array}$ & Texto & Topográfico \\
\hline Winslow, J. & $\begin{array}{l}\text { Exposition anatomique de } 1 \text { a } \\
\text { structure du corps humain }\end{array}$ & París, Francia & Francés & 1732 & Tratado & Descriptivo \\
\hline
\end{tabular}

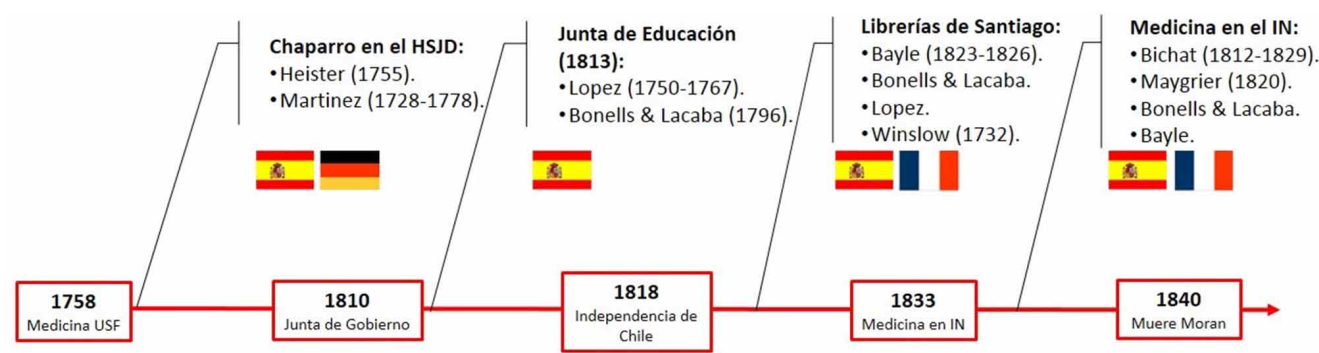

Fig. 7. Línea de tiempo que relaciona los libros de la bibliografía anatómica con el contexto histórico chileno, considerando su aparición en la literatura especializada (las banderas solo fueron incluidas como imágenes referenciales y no necesariamente corresponden al periodo histórico en cuestión).

\begin{tabular}{lcccc}
\hline Texto & P & p & Pxp & ICI(c) \\
\hline Bayle, A. & 512 & 280 & 143360 & 0,4946 \\
Bichat, X.; Buisson, M.; Roux, P. & 2609 & 242 & 631378 & 2,1785 \\
Bonells, J.; Lacaba, I. & 1180 & 441 & 520380 & 1,7955 \\
Heister, L. & 238 & 336 & 79968 & 0,2759 \\
Lopez, J. & 1019 & 171 & 174249 & 0,6012 \\
Martinez, M. & 600 & 341 & 204600 & 0,7059 \\
Maygrier, J. & 538 & 372 & 200136 & 0,6905 \\
Winslow, J. & 740 & 506 & 374440 & 1,2920 \\
\hline
\end{tabular}

Tabla II. Tabla que resume los resultados del análisis cuantitativo de los libros de textos utilizados en la formación anatómica de médicos en Chile entre 1758 - 1840. P: paginas totales del libro de texto. p: promedio simple de palabras por páginas. Pxp: paginas totales por el promedio simple de palabras por páginas. ICI(c): índice de cantidad de información comparado de la obra analizada. 
La Figura 7, permite observar la relación de los textos con los acontecimientos históricos relevantes de este periodo en Chile. Así podemos observar como durante el periodo en que este país es colonia española (hasta 1818), los textos que priman son preferentemente de autores españoles (Bonells \& Lacaba; Martínez; Lopez) y el único texto que no es español (Heister), fue traducido e incorporado por un cirujano español. Posterior a la Declaración de Independencia de Chile (1818), se incorporan un número importante de obras de origen francés, lo que denota el ambiente social e histórico que viven las antiguas colonias españolas en América.

Del análisis cuantitativo: Posterior a haber realizado el análisis de ICI(c), podemos indicar que existen libros que sobrepasan la cantidad de información que se determinó como el estándar de comparación (índice MD). Estos textos que poseen un ICI(c) mayor a 1.0 corresponden a los textos de gran extensión o tratados (Bichat et al; de Bonells \& Lacaba; Winslow). Otros tres libros quedan con un ICI(c) menor a 1.0 pero mayor a 0.5 , clasificándose como textos de mediana extensión (Lopez; Martínez; Maygrier. Finalmente, dos son los textos que presentan un ICI(c) menor a 0.5 y que por su pequeña extensión, bien tienen al llamarse compendios (Bayle; Heister).

Es indudable la importancia de los libros de texto que conforman la bibliografía de un curso universitario, no solo entregan el acervo necesario para adquirir el conocimiento y las competencias específicas de la asignatura, sino que también determinan la orientación y el contexto histórico, político y social del momento. El estudio profundo de los orígenes de la formación de médicos en Chile es fundamental para comprender el nacimiento de la identidad profesional, que ha sido de gran influencia en las ciencias de la salud y en la historia político - social de Chile. Este trabajo representa un aporte importante a la comprensión del origen de la formación de esta profesión, entregando información sobre la bibliografía que estos primeros estudiantes revisaron, estudiaron y asimilaron, permitiendo conformar la visión del cuerpo humano sano y enfermo a través de la enseñanza de la anatomía humana.

SILVA, J.; ARAYA, C.; CARDENAS, J.; SALCEDO, A.; MANSILLA, B.; HERNÁNDEZ, A.; RODRÍGUEZ, P.; SALAZAR, M.; CARACUEL, R. \& ESPINOZA, M. Anatomical literature used in the training of physicians in Chile between 1758 and 1840. Int. J. Morphol., 37(3):938-946, 2019.

SUMMARY: The study of the teaching of medicine, from a historical perspective, is important to explain the development of this profession. Considering the value of books in the university curriculum, its study allows us to understand the socio-political influence of the historical moment. The objective of this work was to determine and analyze the first bibliography used in the training of doctors in Chile between 1758 and 1840 (from the foundation of the University of San Felipe to the death of Dr. Pedro Morán).Through a historical investigation it was possible to find the eight textbooks used during this period. Of these, a descriptive analysis was carried out that included the identification of the author, the title of the work, the year, language and place of publication. In addition, they were classified according to extension and focus. A quantitative analysis was also carried out to determine the amount of information presented in these books compared to modern literature. The results were arranged in tables and graphs for later analysis. The texts found were mainly published in Spain and in Spanish $(62.5 \%)$, although an important part was published in France and in French (37.5\%). The bibliography of French authors was used mainly (50\%), although books by Spanish authors (37.5 $\%)$ and Germans $(12.5 \%)$ were also included. With respect to the extension of the texts, both the medium and large extension books were represented in the same way $(37.5 \%)$ and the compendiums represented a smaller percentage $(12.5 \%)$. This work is a contribution to the understanding of the origin of the training of doctors in Chile and helps to understand the birth of professional identity, which has been of great influence in the political - social history of Chile, describing the bibliography that marked the birth of the History of Anatomy in our country.

KEY WORDS: History of anatomy; History of medicine; History of Chile; Anatomical literature; University of San Felipe; National Institute.

\section{REFERENCIAS BIBLIOGRÁFICAS}

Amunategui, D. Los Primeros Años del Instituto Nacional (1813 - 1835). Santiago de Chile, Imprenta Cervantes, 1889.

Barros-Arana, D. Historia General de Chile. $2^{\mathrm{a}}$ ed. Santiago de Chile, Universitaria, 2001.

Campos, F. Desarrollo Educacional 1810 - 1960. Santiago de Chile, Editorial Andrés Bello, 1960.

Cárdenas, V. J. L. History of anatomy in Chile. The beginnings. Int. J. Morphol., 35(3):958-69, 2017.

Cruz-Coke, R. Historia de la Medicina Chilena. Santiago de Chile, Editorial Andrés Bello, 1995.

Ferrer, P. Historia General de la Medicina en Chile. Desde El Descubrimiento y Conquista de Chile en 1535, Hasta Nuestros Días. Talca, Imprenta Talca, 1904.

Flores, N. Historia de la Anatomía en Chile. Arch. Chil. Morfol., 1:7-25, 1933.

Gay, C. Historia Física y Política de Chile (Reimpresión). Santiago de Chile, Centro de Investigaciones Diego Barros Arana de la Dirección de Bibliotecas, Archivos y Museos, 2007.

Grossi, J. Reseña del Progreso Médico en Chile. Valparaíso, Imprenta de La Opinión, 1895.

Hernández, J. La Universidad de Chile. Santiago de Chile, Ediciones Universidad de Chile, 1934.

Laval, E. \& Duarte, I. Enseñanza de la medicina en Chile colonial durante el siglo XVIII. El catedrático Domingo Nevin y su alumno Pedro Manuel Chaparro. Rev. Chil. Infectol., 33(5):565-9, 2016.

Laval, E. El doctor Pedro Morán, la Escuela médica de 1833 y la batalla de Rancagua. Rev. Chil. Infectol., 28(5):484-7, 2011. 
Laval, E. Evolución y desarrollo de la enseñanza de la Anatomía en Chile. An. Hist. Med., 6(2):7-75, 1964.

Laval, E. Francisco Julio Lafargue y Carlos Buston: el episodio de "la mano femenina". Rev. Chil. Infectol., 19(6):682-4, 2012.

Laval, E. Vida y Obra de Fray Pedro Manuel Chaparro. Medico Chileno del Siglo XVIII. Santiago de Chile, Editorial Universidad Católica, 1958.

Medina, J. La medicina y los médicos de la Real Universidad de San Felipe. Santiago de Chile, Soc. Imp. y Lit. Universo, 1928.

Mellafe, R.; Rebolledo, A. \& Cárdenas, M. Historia de la Universidad de Chile. Santiago de Chile, Ediciones Universidad de Chile, 1992.

Orrego, A. Recuerdos de la Escuela. Santiago de Chile, Editorial del Pacifico, 1922.

Salas, E. Historia de la Medicina en Chile. Con Importantes Documentos sobre la Medicina de Nuestros Predecesores. Santiago de Chile, Imprenta Vicuña Mackenna, 1894

Semir, M. Apuntes para la historia de la enseñanza de la medicina en Chile. An. Univ. Chile, 17(8):737-56, 1860

Serrano, S. Universidad y Nación. Chile en el Siglo XIX. Santiago de Chile, Universitaria, 2016

Sierra, L. Cien años de enseñanza de la medicina en Chile. An. Fac. Biol. Cienc. Med., 1:3-353, 1934.
Dirección para correspondencia:

Prof. Dr. Juan Luis Silva R.

Académico - Investigador

Facultad de Medicina

Universidad San Sebastian

Campus Los Leones de Providencia

Dirección Lota 2465, Edificio F

Santiago

CHILE

Email: juan.silva@uss.cl

Recibido: 21-12-2018

Aceptado: 07-02-2019 